\title{
Adaptive Predictive and Recommendation System Based on Learners Style
}

\author{
S.Jayaprakash ${ }^{1}$, S.Vishnupriya ${ }^{2}$, Dr.R.Kumar ${ }^{3}$ \\ ${ }^{1}$ M.E CSE, Roever Engineering College, Perambalur, Tamilnadu, India \\ ${ }^{2}$ Department of Information Technology, Assistant Professor, Engineering College, Perambalur, Tamilnadu, India \\ ${ }^{3}$ Department of Information Technology, Professor, Engineering College, Perambalur, Tamilnadu, India \\ Email: jpcse014@gmail.com ${ }^{1}$ svn.vishnupriya@gmail.com² rkumarnkl@gmail.com ${ }^{3}$
}

\begin{abstract}
E-Learning development has been a widely adopted methodology nowadays. But there are main risks for learners that they cannot understand in a single stretch of learning. The learning style of every learner varies according to the knowledge and learning object level. Several points have emerged regarding the conception, development and maintenance of E-learning solutions. In our proposed system, Felder Silverman learning style modules had been used to analyze the learner style and the predictive mechanism is enhanced using machine learning methods. The risk of learner will be reduced with the implemented tool. Deep learning can predict the learner style trained labeled data inputs and the tested outputs produce a visualization model of the learner score. The learner scores and the recommendation strategy are added to make learning easier using E-learning technology. Thus the learners can be known as (visual, aural, physical, logical, etc.). This reduces the risk of online learners and the learning objective is well known by the user itself using the implemented methodology. System act as an instructor with the proposed system and an artificial intelligence system is added for a systembased instructor. The statistical test showed that both the behavioral intervention and recommending intervention is based on earlier prediction played a positive role and learning engagement.
\end{abstract}

Key terms - Felder Silverman learning style, Deep learning, recommendation strategy, behavioral intervention.

\section{INTRODUCTION}

Analyzing the user behavior with the interacting learning environment and challenging the area's target enhanced in learning. Various learning environments are provided such as adaptive learning environment, collaborative learning environment, course management system, and intelligent tutoring system has been here. Each learner's current states vary with this environment and the knowledge creditability that they have been widely adapted. The implemented learner style adaption and the behavior may vary with each learner. Identification of the learner style on each learner is done through the psychological fact. Deep learning and interactive learning technology is widely used for the learner module and the objective classification. Still now two major open issues exist in the E-Learning environment one: risk of nonunderstanding the notes and the PDFs provided by the website providers and two: varying constants of learner objectives are still now could not be understood by the learner itself. So a personnel interaction skill with the adaptive learning and the deep learning process with an interactive purpose enhance the most comfortable model of identification and the learning objectives vary on each user.

The system widely checks using the Felder Silverman modeling technique such that the user learning models are matched with the training models. The interactive data set is added with the machine learning and artificial intelligent approach to make the system prediction. However, the learner model's collection in the first module and then the learner style visualization make a good interface for the user and the website provider. The interaction protocols and the heterogeneous data set can be identified and picked for the data set, which is all added. The collection of these labeled data should be based on the learner environment and the selection of each option is filled with the scores and the performance is analyzed based on the scores gathered.

This paper's contribution mainly focused on early risk prediction of the online courses and smart E-Learning system. Previous work has been performed on the various analysis made as the feature extraction and data mining algorithms. Thus the behavioral intervention system had developed for recent advancement. 
The rest of the paper is organized as followed chapters. Chapter 2 contains a literature survey of the reviewed paper. The proposed methodology is organized in chapter 3 and the algorithm is clearly explained in chapter 4. The performance gets analyzed and the issues of the existing system get overcame in chapter 5 .

\section{RELATED WORKS}

(i) Constructing interpretive views of learners: Learners style model with various raw collecting data is added with the system. Each learner's model may vary with the learner model and the instructor provided with peers. An empirical study on each student is made. The evidence of understandability and expressiveness is proved with the analysis of each cognitive skill. Kyparissia A. Papanikolaou has proposed externalizing learners behavior and interaction support may indicate visualization.

(ii) E- Learning challenges and research opportunities: Machine Learning (ML) and Data Analytic (DA) based raw data collection is adapted in this system. The valuable patterns with the collected information are get extracted in this system. Brief study is made about the E-learning systems, challenges and approaches are well identified by the data mining and deep learning technologies. The research exploits many areas and implementations to be added to the system.

(iii) Learners influence propagation: The learner's interpersonal information is getting the collaborative filtering method and hybrid filtering to combine the learner's influence model. The learners' recommendation is propagated in this system so that the Learners Objective is well known by the following information: Learners Influence Model (LIM) proposes the independent ratings of learner's similarity. Intutionistic Fuzzy Logic (IFL) is applied for the accuracy prediction of LIM. Such the experimental demonstration adds the diversified recommendations and the adaptability of learners to the Learning scenario.

(iv) Pedagogical interventions of learning engagements: The process added with the click stream user behavior identification system. The student performance is analyzed by diverse set of functions. Tradaboost transfer learning method is added with the former course interaction. The teaching iterations and its high precision is identified in this network. The trained model helped the timely intervention of the positive role prediction.

(v) A predictive model for at-risk student identification: the study proposes a predictive model identification of the student's performance analysis to address the risk of the learning analytics. Two datasets are collected as the raw

Vol. 4 (5), November 2020, www.ijirase.com datasets by the Jui-Long Hung, Brett E. Shelton, Juan Yang, Xu $\mathrm{Du}$ authors for the higher related education with the education environment. Lack of common predictors is identified using K12 and this system knows misplaced focus.

(vi) Identifying risk using 100 case studies: The student's performance is identified by three machine learning algorithms: instance-based learning classifier, decision tree, and naïve bayes. This system investigates the risk of the student while learning. The combined result of the machine learning algorithm classifies the student performance. The process is done using machine learning mechanism the author carried out in two process training and testing phases. The data set gathered with time-variant and time-invariant attributes are gathered to identify the prediction accuracy. But the performance measurement can only be known using the added system. The evaluated terms are sensitivity and accuracy prediction with the precision time.

(vii) Development of learning solutions: An E- learningbased software development is achieved with the emerged several points. The automated approaches are made combined with different methodologies of human and social aspects. These points of view support several aspects. This facilitates common E-learning applications and solutions by multidisciplinary teams of software development. Here efficient software is developed for good learning.

(viii) Performance Prediction using machine learning: Havan Agarwal proposed a machine learning-based Neural Network to predict student performance. Different attributes and features are considered in order to determine student performance. The power and accuracy of Neural networks are well analyzed in this paper.

(ix) Early Warning drop in high school: Potential early warning drop in school studies are predicted in advance. The predictions are made from165,715 students who are connected with the Ministry of Education in Korea. The predictive model shows excellent performance and binary classification of students drop out. Here random forest models evaluate big data education. The predictions are made using a data set analysis.

(x) Learner style using Item Response Theory (IRT): Analyzing learner style at the beginning of the course is explained. The learning models and the learning style is identified using Item Response Theory with a personalized learning and high satisfaction of engaged learners.

\section{LEARNER STYLE PREDICTION}

Learning style prediction based on pattern based prediction method. The effectiveness of prediction is calculated using 
Web Usage Mining (WUM), where the mining is done using the E-learning portal. The learner's navigation is studied with the identified patterns of WUM and the efficiency of interface components, with the customizing resource delivery. The student profile is first added with new characteristics and dimensions. The first processing can be delivered as that the learner is a newcomer or old user. The process can be carried out only for the newcomer and the application founds out using the registration process. From the profile built the data collection is made and the behavior gets analyzed on the data set added as questions to the user. The primary research done here is a set of students who were undergoing a course using a website. The comparative study of the existing system and the proposed system is made with 134 students in our system.

Assume the set of students as followed:

$$
S=\left\{s_{i}^{j} \mid, s_{1}{ }^{j}, s_{2}{ }^{j}, \ldots \ldots . . . s_{n}{ }^{j}\right\}
$$

The students were classified by age and the learning style and understanding capacity. The level of knowledge creditability varies according to the period. So we have chosen five different age limit of students which includes lesser than 17 years old18-24 years old, 25-34 years old, 35-54 years old and over 55 years old. The age attribute is also found out because to find the knowledge similarity and creditability over varied age. This seems to be an essential parameter to determine the degree of focus on learning. According to behavioral psychology, data profiles and questions will help analyze the student's behavioral style. The deep understanding and machine learning analysis is made as follows: (1) Students are classified as newcomer and old user, (2) old user will be already categorized, but the new user will be provided with general knowledge objective questions (3) the data training process will be added with a score technology (4) the learner style will be analyzed with the score added with the training implementation. (5) A recommendation system is added according to the user learning behavior identified. (6) Now the performance is evaluated for the proposed system compared to the existing system.

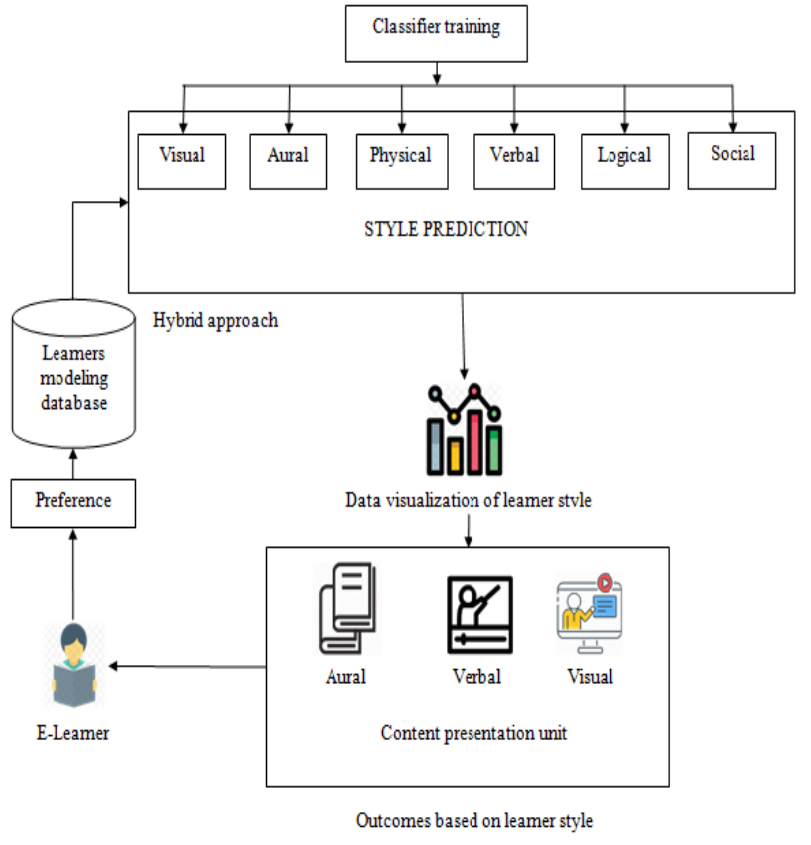

Fig 1 System architecture of learning recommendation system

The data collected from the learner will be known as the preference dataset, where they are Self Obligation personalities (SOP). The self-assessment system and the recommendation system will be based on the content and the observation grain (i.e), the precision of the events considered as units in the analysis. Multiple levels of meaningful hierarchy are attached to the domain model. The dataset can be predicted as per the training is made for the questions. The interaction system is made with navigational and temporal data with many types of resources. Such that the variables and the considered context variables identify the particular environment with specific affordances.

\section{FELDER SILVERMAN STYLE PREDICTION}

The learning style form is inspired by the Felder Silverman learning style model, which provides a good analysis of learning style prediction. Four dimensions can analyze the implementation. First, the dimensions can be active and reflective style, which shows the learner's personality traits. Secondly, it distinguishes the sensing and intuitive learning style, and the third is a visual-verbal dimension and the fourth sequential learning styles. The scores for each dimension are calculated according to the question they determined. The scores are calculated as follows: 
(i) Visual (7)

(ii) Verbal (6)

(iii) Logical (5)

(iv) Aural (4)

(v) Physical (3)

(vi) Social (2)

(vii) Solitary (1)

These values are organized as the extracted features from the reference dataset and the knowledge level is identified. To get the psychological factors, intuitionistic fuzzy logic is matched with the level of score that the student gains. The knowledge uncertainty of a learner will be represented through the membership and non-membership values. The determination of the student behavior is done by neuroticism, extraversion, agreeableness and conscientiousness.

\section{RECOMMENDATION SYSTEM}

Recommendation systems are based on machine learning and information retrieval system. The recommendation system is the potential need in this system, which produces the educational resource according to the user interest. Most of the academic learning system provides help to all the learners as same. The implemented suggestion system suggests contents for E-learning users according to the predicted behavior. Somehow recommendation system will also suggest $\mathrm{CD}$, Books for the user preference choice. In general, to enable personalization and the existing system use of one or more knowledge involve adaptive course delivery. Here the system acts as the tutor for the students and an artificial approach is well implemented. Adaptive collaboration support and adaptive interaction ensure the system's real-time requirement, which did research proposed on different algorithms. User-based collaborative filtering filters the learner according to the user behavioral prediction. The system act with a dynamic interface since static prediction will not efficiently give the right output. A real-time user preference has been enhanced, and the future predictions come up with the relative information on the past course chosen. After which, the online-based test is allotted on each learner and the analysis is made.

\section{PERFORMANCE EVALUATION}

The performance evaluation of the implemented system is done with the accuracy of the predicted system. The researchers are made over 56 students. As mentioned above, the learners are classified on age basis attributes and the variations are analyzed. The interpreting visualization shows the learner behavior and a recommendation system with the analysis of learner behavior. The implemented study here focused on

- Which evaluation criteria the collaborative analyses are made?

- How do learners visualize interpretations and found out the learning style using this system?

Integration protocol of evaluating learner visualization:

Example 1: What do you think when you hear the word cat?

Option 1: Remember the word of the cat

Option 2: Remember the cat picture where I will make it in my brain

Option 3: The cat sounds meow is embracing

Here three options has a score where options A will be aural (), option 2 will be Visual () and option 3 is logical (). The learner score according to the option they choose. The process gets compared with the trained data set and makes the entire behavioral system. Thus the system can be highly processed in the implemented way.

Table 1 scores of learning style

\begin{tabular}{|l|l|l|}
\hline S. No & Learning style & Score \\
\hline 1. & Visual & 7 \\
\hline 2. & Verbal & 6 \\
\hline 3. & Logical & 5 \\
\hline 4. & Aural & 4 \\
\hline 5. & Physical & 3 \\
\hline 6. & Social & 2 \\
\hline 7. & Solitary & 1 \\
\hline
\end{tabular}

\section{Visualizing component:}

The analyzer's visualization component has displayed the comes about; it reflects the effectiveness of instructing techniques. The visualization component to reflects whether the course embraces well to the diversified learning styles of the understudies. Visualization modes are partitioned into two categories i.e., cohort mode and standard mode. The common mode gives an elevated level of the bolster for different learning strategies or styles. The common-mode adopts the Felder-Silverman learning styles show (FSLSM) approach. These learning styles are delineated as a rate, which is calculated by the average of the three variables. A score illustrates no back for a specific learning style; though, a one $100 \%$ would demonstrate total back. The higher level of the bar represents that the understudies are satisfied with the bolster level. 


\section{CONCLUSION AND FUTURE ENHANCEMENT}

This system has illustrated the course analyzer system's legitimacy at the cohort level and sheds light on how the course can be moved forward. The instrument can be accommodating for teachers in assessing the inclination of understudies with respect to a specific course, to this conclusion, the teaching technique can be significantly progressed driving to superior students' evaluation results. In addition, the system will moreover offer assistance educates to create great communication level with understudies by too considering their choices. The apparatus can be utilized by instructors at all instruction levels. The teachers can utilize the system by giving course material on a specific instrument. In this way, through this method, understudies will pick up knowledge regarding the course without any disaster. In addition, talks between educator and student will be progressed, as the device makes difference instructors to analyze the discernments of understudies, effectively. Besides, the concentrated of input and the criteria of assignments can too be significantly made strides.

In spite of the fact that, the pilot course was not able to supply a suitable learning environment for either detecting or visual learners; in any case, it illustrated the real-world achievability of the intelligently apparatus. The free and dependent cognitive styles come about in fruitful models that attempt to get it their learners. In the present ponder, the course analyzer instrument based on learning styles has primarily centered on the teacher's consideration regarding the potential modifications within the course structure (e.g., adding learning objects) and move forward the bolster the course given for understudies with different learning styles. It has potential to not as it were make strides the plan of the course in future but too permit more understanding into generally understudy execution and address issues specified in past ponders. The suggestions of having such a device are various, with the capacity to decide specific qualities and shortcomings of a course from the pedagogical point of view. It is also believed that teachers will be able to use this tool to make changes not only post hoc but also during the course itself.

\section{REFERENCES}

[1] Papanikolaou, K. A. (2014). Constructing interpretative views of learners' interaction behavior in an open learner model. IEEE Transactions on Learning Technologies, 8(2), 201-214.

[2] Moubayed, A., Injadat, M., Nassif, A. B., Lutfiyya, H., \& Shami, A. (2018). E-learning: Challenges and research opportunities using machine learning \& Data analytics. IEEE Access, 6, 3911739138.

[3] Wan, H., Liu, K., Yu, Q., \& Gao, X. (2019). Pedagogical Intervention Practices: Improving Learning Engagement Based on Early Prediction. IEEE Transactions on Learning Technologies, 12(2), 278-289.

[4] Hung, J. L., Shelton, B. E., Yang, J., \& Du, X. (2019). Improving predictive modeling for at-risk student identification: A multistage approach. IEEE Transactions on Learning Technologies, 12(2), 148-157.

[5] Er, E. (2012). Identifying at-risk students using machine learning techniques: A case study with is 100. International Journal of Machine Learning and Computing, 2(4), 476.

[6] Dodero, J. M., García-Peñalvo, F. J., González, C., Moreno-Ger, P., Redondo, M. A., Sarasa-Cabezuelo, A., \& Sierra, J. L. (2014). Development of e-learning solutions: Different approaches, a common mission. IEEE Revista Iberoamericana de Tecnologias del Aprendizaje, 9(2), 72-80.

[7] Wan, S., \& Niu, Z. (2019). A hybrid e-learning recommendation approach based on learners' influence propagation. IEEE Transactions on Knowledge and Data Engineering, 32(5), 827840.

[8] Agrawal, H., \& Mavani, H. (2015). Student performance prediction using machine learning. International Journal of Engineering Research and Technology, 4(03), 111-113.

[9] Chung, J. Y., \& Lee, S. (2019). Dropout early warning systems for high school students using machine learning. Children and Youth Services Review, 96, 346-353.

[10] Rami, S., Bennani, S., \& Idrissi, M. K. (2017, July). Towards a method for analyzing learning style using item response theory. In 2017 16th International Conference on Information Technology Based Higher Education and Training (ITHET) (pp. 1-5). IEEE. 